\title{
The theory of equilibrium magnetic properties of the granulated magnetic materials
}

\author{
E. Stefanovskii \\ Department of Physics, Ben-Gurion University of the Negev, Beer-Sheva, 84105, Israel \\ E-mail: stefan@bgumail.bgu.ac.il \\ Received August 3, 1999
}

\begin{abstract}
The present-day analysis of the theoretical adaptation status of the experimental curves of the magnetization and the static magnetic susceptibility as the functions of external parameters $\mathbf{H}$ and $T$ ( $\mathbf{H}$ is an applied external magnetic field and $T$ is a temperature) is made for granulated ferromagnetic systems. Once more it is pointed that the consideration of the energy of magnetic anisotropy of a ferromagnetic material (as against the methods of the adapt used everywhere) play an essential role for the understanding of the magnetic behavior of the above systems mentioned and allow us to investigate the magnetoanisotropic behavior of granulated magnetic systems [at the certain granule distribution functions (by their volume and orientation) as regards the external magnetic field]. It is shown that the use of the «blocking» concept is not necessary for the investigations of the thermodynamically equilibrium magnetic properties of the above systems.
\end{abstract}

PACS: $75.30 . \mathrm{Gw}, 75.50 . \mathrm{Lk}, 75.50 . \mathrm{Tt}$

\section{Introduction}

Granulated magnetic materials (GMM) have long been subject of intensive and quite successful research. They consist of small ferromagnetic (FM) particles (clusters, granules) in a nonmagnetic (weakly magnetic) matrix. The subject of research can be dielectric materials of the FM component and matrix as well as conducting materials [1-5].

The renewed interest in GMM-materials is explained by the recent observation of the so-called effect of giant magnetoresistance (GMR) in such conducting systems $[6,7]$.

It is not hard to understand that the GMR-effect is connected with an additional channel of current carriers scattering on FM granules and is secondary relating to the unique magnetic properties of GMM materials. Therefore, below, as the first stage of research, we limit ourselves to the analysis of equilibrium magnetic properties of the systems considered above.

\section{Object of research and its main features}

A. The object under investigation is a solid heterogeneous system (an ensemble of FM particles incorporated into a nonmagnetic solid matrix). As a rule, these are the systems with a small (volume) content of the FM component which is essential when interactions between particles of the system are neglected. In other words, FM particles of the GMM-system can neither rotate nor move forward nor change their volume (the state of thermodynamic equilibrium is meant, of course).

B. As is known, the dependence of a magnetic moment or a magnetization (i.e., magnetic moment per unit volume) of a gas of noninteracting paramagnetic (PM) particles on external parameters (absolute temperature $T$ and the value of external magnetic field $H$ ) within the framework of Langevin's classic theory is described by the function of the same name. Thus, magnetization $\mu(H, T)$ of a gas of identical magnetic moments $\mu$ can be expressed as (e.g., see [1])

$$
\mu(H, T)=N|\mu| L\left(\frac{|\mu| H}{k T}\right), \quad L(x)=\operatorname{Coth}(x)-\frac{1}{x},
$$

where $N$ is the number of atomic magnetic moments $\boldsymbol{\mu}$ in the gas unit volume; $k$ is Bolzmann's constant and $L(x)$ is the Langevin function. It is necessary to note that the description of a magnetic particles system with the help of Langevin function (as in the present case) is decisively based on the fact that magnetic energy of each particle of the ensemble possesses only one minimum (in its orientation 
dependence), which corresponds to the orientation of the particle magnetic moment alongside the direction of the applied external magnetic field. It is important that this orientation dependence is described by the function of $\sim \cos \theta$, where $\theta$ is the angle between directions of the magnetic moment and the field.

C. All real FM materials are magnetically anisotropic ones. This means that the magnetic moment of each particle of the ensemble in the absence of external magnetic field tries to orientate alongside the so-called directions of «easy magnetization». For reversing the magnetic moment of each of the ensemble particles from one of these directions to another one (under the influence of some external factors), it is necessary to overcome energy barrier. The value of this barrier is determined by the magnetic anisotropy energy of the particle (and, consequently, by its volume $V$ ) and external factors.

D. If a GMM system consisted of magnetically isotropic FM particles, i.e., if a corresponding energy barrier did not exist, the description of its magnetic properties would be possible with the help of the Langevin function. In so doing the temperature-field dependence of magnetization (e.g., see [1]) of a system-ensemble of identical volume particles in this case is written as:

$$
\mu(H, T)=\mu_{s} L\left(\frac{\mu_{s} V H}{k T}\right),
$$

where $\mu_{s}$ is the saturation magnetization of FM substance, $\mu_{s} V$ is the value of the magnetic moment of the particle with the volume $V$. GMM systems whose magnetization is described by expression (2) (with the addition of «blocking» concept) are usually called superparamagnetics and these concepts were first introduced into the physics of magnetic phenomena by C. Bean [8].

E. Even if, with the help of an external magnetic field in the particles of GMM under investigation the energy barrier connected with the energy of magnetic anisotropy is destroyed, the orientation dependence of magnetic energy of system particles is not described by the $\cos \theta$-function (except the limit case of «a very strong external magnetic field» which will be treated below).

F. Thus it can be stated that the GMM systems with granules of real FM material that we consider cannot be, generally speaking, described by Langevin classic theory. This circumstance was also noted earlier also by L. Néel $[9,10]$, Yu. Reicher, M. Shliomis [11].
G. Nevertheless, up to now, attempts at describing real GMM systems with the help of the Langevin function have continued to be made (see [1-5], for example). However, it should be noted that, in order to describe peculiarities relating to magnetic anisotropy of the FM component, the concepts of «blocking volume» of particles at a fixed temperature and «blocking temperature» of fixed volume of particles were introduced into the physics of magnetic phenomena.

H. In a real GMM system there are FM component particles of different volume, i.e., in the system, there is a certain function of their distribution $f(V)$ by volume $V$. It is evident therewith that the full volume of FM substance in the system is

$$
V_{\text {mag }}=\int_{V_{\min }}^{V_{\max }} V f(V) d V=\sum_{i} f_{i} V_{i},
$$

where $V_{\min }$ and $V_{\max }$ are the minimum and maximum granule volumes existing in the GMM system, $V_{i}$ is the volume of the $i$-granule, $f_{i}$ is the number of granules with volume $V_{i}$. In (3) we gave both continuous and discrete description of the subsystem of FM substance granules in the GMM system.

\section{Magnetic energy of granules, energy barrier and free energy of the system}

The object for investigation is placed in an external magnetic field, the direction of which coincides with that of magnetic anisotropy axes. Neel was the first to consider such a geometry of the problem [10]. At the initial stage of research we neglect the interaction of FM component granules with each other. Moreover, all the particles of the GMM system are supposed to be unidomain, i.e., magnetized homogeneously.

It is evident that, when there is no interaction between particles (and only in this case), the full magnetic energy $\varepsilon_{i}$ of a separate $i$-particle with the volume $V_{i}$ and saturation magnetization of granules of FM substance $\mu_{s}$ represents the sum of a magnetic anisotropy energy $\varepsilon_{a i}$ and its Zeeman energy $\varepsilon_{H i}$ in the external magnetic field, i.e.,

$$
\varepsilon_{i}=\varepsilon_{a i}+\varepsilon_{H i}
$$

As is known, the expression

$$
\varepsilon_{a i}=-K_{\mathrm{eff}} V_{i}\left(\boldsymbol{\mu}_{i}, \mathbf{e}_{z}\right)^{2}
$$

can be written for magnetic uniaxial particles, where the constant $K_{\text {eff }}$ represents the density of 
effective uniaxial magnetic anisotropy energy, $M_{i}=\mu_{s} V_{i}$ is the value of magnetic moment of $i$-particle of the ensemble, $\mu_{i}, \mathbf{e}_{z}$ are unit vectors along the directions of the magnetic moment $\mathbf{M}_{i}$ and Cartesian axis $z$, along which the magnetic anisotropy axes of all system particles and external magnetic field are oriented. The Zeeman energy of the $i$-particle is

$$
\varepsilon_{H i}=-\mu_{s} V_{i} H\left(\mu_{i}, \mathbf{e}_{z}\right) .
$$

In a spherical system of coordinates with the polar axis $\mathbf{e}_{z}$ we have

$$
\begin{gathered}
\boldsymbol{\mu}_{i}=\left(\cos \varphi_{i} \sin \theta_{i}, \sin \varphi_{i} \sin \theta_{i}, \cos \theta_{i}\right), \\
\mathbf{H}=(0,0, H)
\end{gathered}
$$

and the expression for the full energy of $\varepsilon_{i}$ particle is written as

$$
\begin{gathered}
\varepsilon_{i}=-\Delta \varepsilon_{a i}\left(\cos ^{2} \theta_{i}+2 h \cos \theta_{i}\right), \\
\Delta \varepsilon_{a i}=K_{\mathrm{eff}} V_{i}>0, \quad h=\frac{\mu_{s} H}{2 K_{\mathrm{eff}}}=\frac{H}{H_{a}} .
\end{gathered}
$$

The value of $\Delta \varepsilon_{a i}$ determines the energy barrier in the absence of the external magnetic field that is to be overcome in order to change the orientation of magnetic moment $\mathbf{M}_{i}$ from one of the «easy» directions $\theta_{i}=0, \pi$ to another $\theta_{i}=\pi, 0$.

It is not difficult to show that the value of the energy barrier in the external magnetic field of selected orientation decreases with the increase of $h$ value by the law

$$
\Delta \varepsilon_{i}=\Delta \varepsilon_{a i}(1-h)^{2} .
$$

At last, when the value of the external magnetic field $H$ reaches the value of the so-called magnetic anisotropy field $H_{a}$, i.e., $h=1$, the energy barrier disappears [see (10)] and in the energy of the particle $\varepsilon_{i}$ just one minimum is left at $\theta_{i}=0$, i.e., the only one equilibrium state. In this external magnetic field, the energy of the particle $\varepsilon_{i}$ is

$$
\varepsilon_{i}=-\Delta \varepsilon_{a i}\left[(\cos \theta+1)^{2}-1\right] .
$$

While the value of the external magnetic field increases further, there is only additional deepening of the single minimum in the particle energy $\varepsilon_{i}$ at $\theta_{i}=0$.

Based on the above uncomplicated arguments, the following conclusions (very important ones for further discussion) can be made:

1. Granules of different volumes have different values of corresponding energy barriers. This makes it possible, if needed, to introduce the concepts of «volume» and «temperature» of blocking.

2. Energy barriers in the granules of different volume disappear in one and the same external magnetic field which is equal to the field of magnetic anisotropy $H_{a}$ [condition $h=1$ from (9)].

3. Orientational dependence of the energy of $i$-particle $\varepsilon_{i}$, even at energy barrier disappearance, is rather far from being $\sim \cos \theta$ [see (10)]. In other words, even in this case, generally speaking, the Langevin description of the GMM system does not seem to be correct, although all the particles of the system are «unblocking», i.e., they behave in a way resembling a superparamagnetic one.

4. Only in cases (which will be demonstrated below) $T \gg>\max \left\{\Delta \varepsilon_{a i}\right\}$ and $H \gg>H_{a}$ can one use the Langevin description of the system without doubt.

Thus, the energy of the $i$-particle of the GMM system is described by expression (8). The granule ensemble contains $f_{i}$ particles with $V_{i}$ volume, where $f_{i}$ is the function of granules distribution by volume and the full volume $V_{\text {mag }}$ of the FM component is (3). The full energy of the GMM system (the energy of noninteracting granules ensemble) can be written as

$$
E=\sum_{i} f_{i} \varepsilon_{i},
$$

where $\varepsilon_{i}$ is determined by expression (8).

At finite temperatures the probability of detecting the system (having thermodynamic equilibrium) with energy $E\left(x_{i}\right)$ in the volume element of generalized coordinates
Bolzman exponent

$$
\exp \left[-E\left(x_{i}\right) / k T\right] \text {. }
$$

In our case, it means that the probability for the $i$-particle (granule) with volume $V_{i}$ to have the direction of magnetic moment $\mathbf{M}_{i}$ in the element of space angle $d \Omega_{i}=\sin \theta_{i} d \varphi_{i} d \theta$ is

$$
d \omega_{i}=A_{i} \exp \left[\frac{\Delta \varepsilon_{a i}}{k T}\left(\cos ^{2} \theta_{i}+2 h \cos \theta_{i}\right)\right] d \Omega_{i},
$$

where $A_{i}$ is some constant. It is clear that in this case the corresponding statistical sum $Z$ of the system is determined by an expression

$$
Z=\prod_{i}\left\{A_{i} \int_{\Omega_{i}} d \Omega_{i} \exp \left[\frac{\Delta \varepsilon_{a i}}{k T}\left(\cos ^{2} \theta_{i}+2 h \cos \theta_{i}\right)\right]\right\}^{f_{i}}
$$


and the free energy $F$ of the system

$$
F=-T \ln Z
$$

takes the form:

$$
F(H, T)=\sum_{i} f_{i} F_{i}(H, T),
$$

where

$$
\begin{gathered}
F_{i}(H, T)= \\
=-T \ln \left\{A_{i} \int_{\Omega_{i}} d \Omega_{i} \exp \left[\frac{\Delta \varepsilon_{a i}}{k T}\left(\cos ^{2} \theta_{i}+2 h \cos \theta_{i}\right)\right]\right\}
\end{gathered}
$$

It is necessary to emphasize that additivity of free energy $F(16)$ of the system (and the expression for statistical sum $Z$ (14) as the product) is conditioned by our neglecting of FM granule interactions with each other. As will be seen below, the magnetic moment of the system will also possess the additivity property. It is mathematically reflected in the independence of some direction of $i$-cluster magnetic moment from the state of other system particles.

\section{Magnetic moments of system and granules, its constituents, magnetization and static magnetic susceptibility}

As is known, the magnetic moment of $\mathbf{M}(H, T)$ of the system at temperature differing from absolute zero is expressed by

$$
\mathbf{M}(H, T)=-\partial F(H, T) / \partial \mathbf{H},
$$

or, accounting for the geometry and symmetry of our problem,

$$
\begin{gathered}
\mathbf{M}(H, T)=M(H, T) \mathbf{e}_{z}, \\
M(H, T)=-\partial F(H, T) / \partial H .
\end{gathered}
$$

Taking into account expression (16) for the system free energy, it is easy to obtain from (18)

$$
\begin{gathered}
M(H, T)=\sum_{i} f_{i} M_{i}(H, T), \\
M_{i}(H, T)=T \times \\
\times \frac{\partial}{\partial H} \ln \left\{A_{i} \int_{\Omega_{i}} d \Omega_{i} \exp \left[\frac{\Delta \varepsilon_{a i}}{k T}\left(\cos ^{2} \theta_{i}+2 h \cos \theta_{i}\right)\right]\right\}
\end{gathered}
$$

Further on, it will be convenient for us to use the so-called reduced parameters (as regards the internal parameters of the system, namely as regards the value of energy barrier in the absence of external magnetic field and the value of magnetic anisotropy field)

$$
\begin{gathered}
a_{i}^{2}=\Delta \varepsilon_{a i} / k T=K_{\mathrm{eff}} V_{i} / k T, \quad h=H / H_{a}, \\
H_{a}=2 K_{\mathrm{eff}} / \mu_{s} .
\end{gathered}
$$

This is convenient because, e.g., for GMM systems with various FM components (i.e., different values of $K_{\text {eff }}$ and $\mu_{s}$ ) but with identical functions of particles distribution by their volume $f(V)$ and identical $V_{\text {mag }}$ and proportion $K_{\text {eff }} V_{0} / k T$, where $V_{0}$ is the position of function maximum $V f(V)$, the curves of reduced magnetization

$$
m\left(a_{i}^{2}, h\right)=\left(\sum_{i} f_{i} M_{i}(H, T)\right)\left(\mu_{s} \sum_{i} f_{i} V_{i}\right)^{-1}
$$

as functions of reduced magnetic field $h$ just coincide.

It is not difficult to show that, when the variables (20) are used, the expression for the system magnetic moment (19) can be written as

$$
\begin{gathered}
M(H, T)=\sum_{i} f_{i} M_{i}\left(a_{i}^{2}, h\right) \\
M_{i}\left(a_{i}^{2}, h\right)= \\
=\frac{a_{i}^{2}}{2}\left\{\int_{\Omega_{i}} d \Omega_{i} \exp \left[a_{i}^{2}\left(\cos ^{2} \theta_{i}+2 h \cos \theta_{i}\right)\right]\right\}^{-1} \times \\
\times \frac{\partial}{\partial h} \int_{\Omega_{i}} d \Omega_{i} \exp \left[a_{i}^{2}\left(\cos ^{2} \theta_{i}+2 h \cos \theta_{i}\right)\right]
\end{gathered}
$$

In the case of continuum description

$$
\begin{aligned}
& m(H, T)= \\
& =\left(\int_{V_{\min }}^{V_{\max }} V f(V) \Phi\left[a^{2}(V), h\right] d V\right)\left(\int_{V_{\min }}^{V_{\max }} V f(V) d V\right)^{-1} \\
& \text { where } m(H, T) \text { is the reduced magnetization } \\
& (|m(H, T) \leq 1|) \text {, and }
\end{aligned}
$$




$$
\begin{gathered}
\Phi\left(a_{i}^{2}, h\right)= \\
=\frac{\exp \left[a_{i}^{2}(1+h)^{2}\right]-\exp \left[a_{i}^{2}(1-h)^{2}\right]}{\sqrt{\pi} a_{i}\left\{\operatorname{erfi}\left[a_{i}(1+h)\right]+\operatorname{erfi}\left[a_{i}(1-h)\right]\right\}}-h= \\
=\frac{1-\exp \left(-4 a_{i}^{2} h\right)}{2 a_{i}\left\{F_{D}\left[a_{i}(1+h)\right]+\exp \left(-4 a_{i}^{2} h\right) F_{D}\left[a_{i}(1-h)\right]\right\}}-h .
\end{gathered}
$$

In expression (24) for the function $\Phi\left(a_{i}^{2}, h\right)$ both special functions

$$
\operatorname{erfi}(x)=\frac{2}{\sqrt{\pi}} \int_{0}^{x} d t \exp \left(t^{2}\right)
$$

and the Dawson integral were used

$$
F_{D}(x)=\exp \left(-x^{2}\right) \int_{0}^{x} d t \exp \left(t^{2}\right) .
$$

Thus, from expressions (23)-(26) it is clear that equilibrium curves of magnetization of a real GMM system consisting of magnetic anisotropic FM material granules (in this case of magneto-uniaxial), and, consequently, all equilibrium magnetic parameters of such a system in the whole range of external parameters change ( $H$ and $T$ ) can be described only by the $\Phi\left(a_{i}^{2}, h\right)$-function and cannot described by the Langevin function $L\left(2 a_{i}^{2} h\right)=$ $=L\left(\mu_{s} V_{i} H / k T\right)$.

There are quite substantial reasons for believing that in real GMM systems the so-called LifshitzSlyozov-Wagner logarithmically normal (log-normal) distribution $[12,13]$ of particles by volume is implemented (see, e.g., [12-14]), i.e.,

$$
f(V)=\frac{1}{\sqrt{2 \pi} V \sigma} \exp \left\{-\frac{\ln ^{2}\left(V / V_{0}\right)}{2 \sigma^{2}}\right\} .
$$

In expression (27): $\sigma$ is dispersion (half-width) of the corresponding distribution while $V_{0}$ is the volume corresponding to the maximum of the function $V f(V)$. It is necessary to note that the using of the log-normal distribution function (its properties) gives us the possibility of extending the limits of integrations in (23) to the whole interval of volumes $\{0, \infty\}$.

The temperature parameter $a_{i}^{2}$ can be expressed by the so-called blocking temperature. As is known, (see, e.g., [1-5]), the blocking temperature of the particle with $V_{i}$ volume in the absence of external magnetic field is determined as

$$
T_{\mathrm{bl}, i}^{0}=K_{\mathrm{eff}} V_{i} / k \ln \left(\frac{\tau}{\tau_{0}}\right),
$$

where $\tau_{0}$ is relaxation time and $\tau$ is observation time (as a rule, in the usual experiment to measure static magnetic equilibrium properties $\ln \left(\tau / \tau_{0}\right) \approx 25$ is used). Then, it is evident that we have

$$
a_{i}^{2}=\left(T_{\mathrm{bl}, i}^{0} / T\right) \ln \left(\frac{\tau}{\tau_{0}}\right) .
$$

However, the following should be noted. The temperature and volume of blocking are introduced into the physics of GMM systems when their equilibrium properties are described in order to break the sums or integrals in the numerators of expression (24) into two parts, the first of which relates to «blocked» particles, the second parts relates to «unblocked» particles. In so doing, «blocked» particles are described as showing «ferromagnetic» behavior, while «unblocked» ones, with the help of the Langevin function, as showing «superparamagnetic» behavior.

The use of the function $\Phi\left[a_{i}^{2}(V), h\right]$ instead of Langevin's function eliminates all the noted contradictions and complications and we do not think it is necessary to use the concept of blocking (at least when describing thermodynamic equilibrium magnetic properties of the GMM system). The argument of exponent $a_{i}^{2}(1-h)^{2}$ and the argument of functions erfi $\left[a_{i}(1-h)\right]$ and $F_{D}\left[a_{i}(1-h)\right]$ completely takes into account the competition of heat energy with the full magnetic energy of the particle of any volume at any significant parameters $T$ and $H$. This moment is thought to be quite significant and it is connected with the magnetic anisotropy of granules of the FM material. This more realistic description of magnetic properties of GMM systems was proposed by F. G. West [15] in 1961 (see also, [16]) and was forgot afterward.

Static magnetic susceptibility of a system is determined as

$$
\chi(H, T)=\partial M / \partial H
$$

or, taking into account that

$$
\frac{\partial}{\partial H}=\frac{\mu_{s}}{2 K_{\text {eff }}} \frac{\partial}{\partial h},
$$

we obtain from (30) 


$$
\begin{gathered}
\chi(H, T)=\frac{\mu_{s}^{2}}{2 K_{\mathrm{eff}}} \sum_{i} f_{i} V_{i} \Psi\left(a_{i}^{2}, h\right)= \\
=\frac{\mu_{s}^{2}}{2 K_{\mathrm{eff}}} \int_{V_{\min }} V f(V) \Psi\left(a^{2}(V), h\right) d V,
\end{gathered}
$$

where

$$
\begin{gathered}
\Psi\left(a_{i}^{2}, h\right)=\frac{2 a_{i}}{\sqrt{\pi}}(1+h) \times \\
\times \frac{\exp \left[a_{i}^{2}(1+h)^{2}\right]-\exp \left[a_{i}^{2}(1-h)^{2}\right]}{\operatorname{erfi}\left[a_{i}(1+h)\right]+\operatorname{erfi}\left[a_{i}(1-h)\right]}- \\
-\frac{\pi}{8}\left\{\frac{\exp \left[a_{i}^{2}(1+h)^{2}\right]-\exp \left[a_{i}^{2}(1-h)^{2}\right]}{\operatorname{erfi}\left[a_{i}(1+h)\right]+\operatorname{erfi}\left[a_{i}(1-h)\right]}\right\}^{2}-1 .
\end{gathered}
$$

In conclusion of this section we would like to recall that when describing the GMM system as a superparamagnetic one,

$$
\begin{gathered}
M(H, T)=\mu_{s} \sum_{i} f_{i} V_{i} L\left(2 a_{i}^{2} h\right)= \\
V_{\max } \\
=\mu_{s} \int_{V_{\min }} V f(V) L\left(2 a^{2}(V) h\right) d V,
\end{gathered}
$$

and the static magnetic susceptibility

$$
\begin{aligned}
& \chi(H, T)=\frac{\mu_{s}^{2}}{2 K_{\text {eff }}} \sum_{i} f_{i} V_{i} \Xi\left(a_{i}^{2}, h\right)= \\
& =\frac{\mu_{s}^{2}}{2 K_{\text {eff }}} \int_{V_{\min }}^{V_{\max }} f(V) \Xi\left(a^{2}(V), h\right) d V
\end{aligned}
$$

where

$$
2 a_{i}^{2} h=\frac{\mu_{s} V_{i} H}{k T}
$$

the argument of the Langevin function corresponding to the Langevin classic theory, while

$$
\Xi\left(a_{i}^{2}, h\right)=\frac{\sinh \left(2 a_{i}^{2} h\right)-4 a_{i}^{4} h^{2}}{2 a_{i}^{2} h^{2} \sinh \left(2 a_{i}^{2} h\right)} .
$$

It not difficult to show that, for example, in the case when the orientation of the applied external magnetic field is perpendicular to the magnetic anisotropy axis of granules the $\Phi\left(a_{i}, h\right)$-function in expression (23) has the form (this case corresponds to $K_{\text {eff }}<0$ )

$$
\Phi\left(a_{i}, h\right)=\frac{\left\{\exp \left[-a_{i}^{2}(1+h)^{2}\right]-\exp \left[-a_{i}^{2}(1-h)^{2}\right]\right\}}{\sqrt{\pi} a_{i}\left[\operatorname{Erf}\left\{a_{i}(1+h)\right\}+\operatorname{Erf}\left\{a_{i}(1-h)\right\}\right]}+h,
$$

$$
a_{i}^{2}=\frac{\left|K_{\mathrm{eff}}\right| V_{i}}{k T}, \quad h=\frac{\mu_{s} H}{2\left|K_{\mathrm{eff}}\right|}
$$

where $\operatorname{Erf}(x)$ is the error function.

\section{Some special cases. Comparison with Langevin classic theory}

a) GMM system with granules of identical sizes.

Under these conditions, it is evident that in a discrete description of the GMM system there is only one value of $f_{i}$ different from zero that corresponds to the volume of granules $V_{0}$, and it coincides with the total number of granules in the system. When the GMM system is described in continuum, the distribution function of the system of particles by their volume has the form of the Dirac $\delta$-function

$$
f(V) \sim \delta\left(V-V_{0}\right) .
$$

Here, the reduced magnetization $m(H, T)$ of the GMM system has the form

$$
m(H, T)=\Phi\left(a_{0}^{2}, h\right),
$$

and the static magnetic susceptibility

$$
\frac{2 K_{\text {eff }}}{\mu_{s}} \chi(H, T)=\Psi\left(a_{0}^{2}, h\right),
$$

where $a_{0}^{2}=K_{\text {eff }} V_{0} / k T$.

Figures 1-4 (as the examples) presents the results of numerical calculation of curves $m(H, T)$ vs the Langevin parameter $2 a_{i}^{2} h=\mu_{s} V_{0} H / k T$ and curves $\chi(h)$ and $\chi(t)$ for a GMM system consisting of real FM granules of the same size $V_{0}$ at different correlations of external and internal parameters of the system. In general, it is seen clearly that these results differ essentially from those that were obtained with the help of the Langevin function. Especially significant to note is a substantial increase in the initial static magnetic susceptibility of 


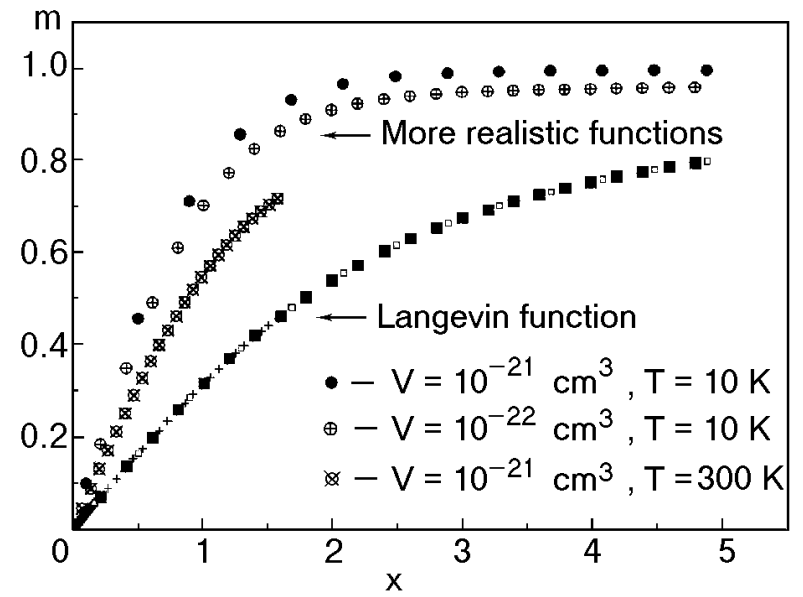

Fig. 1. Dependencies of the magnetization $m=\mu / \mu_{s}$ of GMM system with identical particles on the Langevin parameter $x=\mu_{s} V H / k T$ for different volumes of particles and temperatures $\left(\mu_{s}=10^{3} \mathrm{Gs}, K_{\text {eff }}=10^{8} \mathrm{erg} / \mathrm{cm}^{3}\right)$. The Langevin description of all GMM systems is the same (the different regions of the lowest curve).

the GMM system (as compared to the Langevin one) $\chi(h=0)$ in the range of low temperatures $K_{\text {eff }} V_{0} / k T>>1$. This can be essential when GMM objects are used in devices to record and read out an information.

b) The case $f_{i} V_{i}=$ const ( $V f(V)=$ const $)$ is a specific distribution of the system particles when particles of any size contribute equally to the magnetic component volume of the GMM system.

It is not difficult to show that, in these cases, the reduced magnetization of the system can be expressed in the form

$$
m(H, T)=\frac{1}{V_{\max }-V_{\min }} \int_{V_{\min }}^{V_{\max }} \Phi\left[a^{2}(V), h\right] d V .
$$

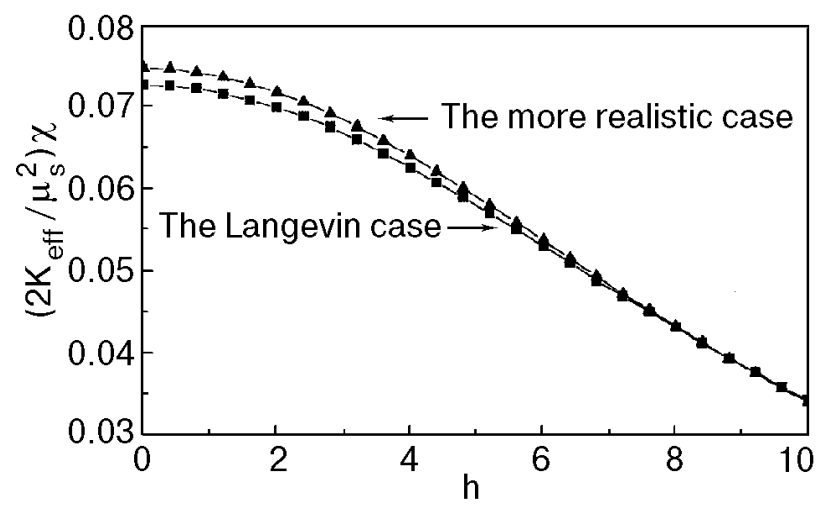

Fig. 2. The static magnetic susceptibility $\chi=d M / d H$ of a GMM system with identical particles as a function of the reduced applied magnetic field $h=H / H_{a}$ at $\ll$ high» temperatures $t=k T / K_{\text {eff }} V=10>>1$.

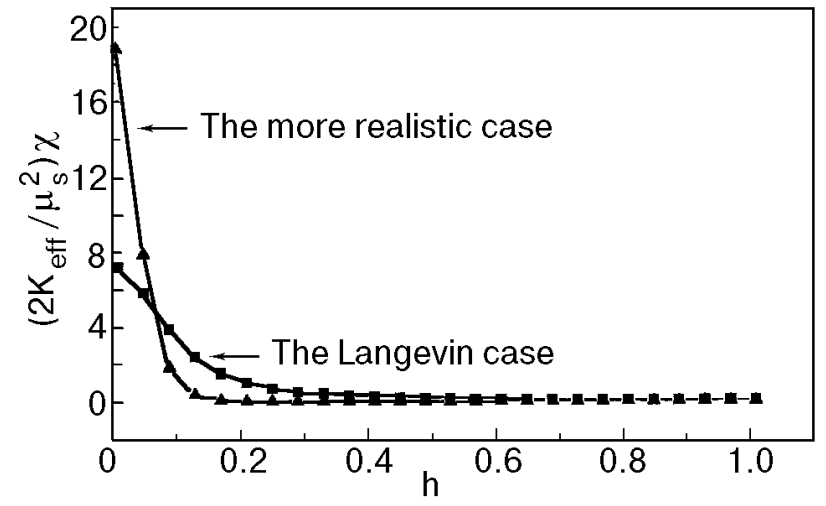

Fig. 3. The static magnetic susceptibility $\chi=d M / d H$ of a GMM system with identical particles as a function of the reduced applied magnetic field $h=H / H_{a}$ at «low» temperatures $t=k T / K_{\text {eff }} V=0.1<<1$.

Using the theorem of integral calculation on the average

$$
\int_{V_{\min }}^{V_{\max }} \Phi\left[a^{2}(V), h\right] d V=\left(V_{\max }-V_{\min }\right) \Phi\left[a^{2}(\hat{V}), h\right]
$$

we come to the expression

$$
m(H, T)=\Phi\left[a^{2}(\hat{V}), h\right]
$$

where $\hat{V}$ is some value of the volume from the range $\left[V_{\min }, V_{\max }\right]$, i.e., $V_{\min } \leq \hat{V} \leq V_{\max }$.

In other words, this GMM system with such a distribution function behaves as a GFM system consisting of identical granules with the volume $V$. Considered below are some limit cases for values of the system's external parameters $H$ and $T$.

c) $H>>H_{a}(h>>1)$.

In these conditions, taking into account that $|\cos \theta| \leq 1$ and neglecting the unity as compared to



Fig. 4. The static magnetic susceptibility $\chi=d M / d H$ of a GMM system with identical particles as a function of the reduced temperature $t=k T / K_{\text {eff }} V$ at «weak» external magnetic field $h=H / H_{a}=0.01<<1$. 
$h$ in expressions (13), (14), (16), (19), (22), we come to the Langevin classic theory. And this is not surprising as it is under these conditions that the energy barrier in all the particles of the GMM system disappears and the orientation dependence of full magnetic energy of the system [see (9)] becomes quite near $\sim \cos \theta$.

The same result can be obtained by using asymptotic expressions for the above mentioned special functions at the corresponding conditions.

d) $h=0$.

In accordance with the general postulates of equilibrium thermodynamics, in this situation, at any temperatures, the system under investigation must be demagnetized, i.e., $m=0$. It is this result that comes out of the expression for the function $\Phi\left(a_{i}^{2}, h\right)$ [see (24)] at $h=0$, as the numerator of the first term is equal to zero, its denominator is equal to $2 \sqrt{\pi}$ erfi $\left(a_{i}\right)$ and the second term is equal to zero.

e) Let us consider now the region of temperatures which are so «high» that one can think that, for $a_{i}$ parameters of all the system particles the conditions $a_{i} \ll<1, a_{i}^{2}<<1$ are fulfilled and the region of values of the external magnetic field is such that $a_{i}^{2} h<<1$ (this corresponds to the case of $\left.\mu_{s} V_{i} H / k T<<1, K_{\mathrm{eff}} V_{i} / k T<<1\right)$. Using expansions

$\exp \left[a_{i}^{2}(1 \pm h)^{2}\right]=1+a_{i}^{2}(1 \pm h)^{2}+\frac{1}{2} a_{i}^{4}(1 \pm h)^{4}+\ldots$

and

$$
\text { erfi }\left[a_{i}(1 \pm h)\right]=a_{i}(1 \pm h)+\frac{1}{3} a_{i}^{3}(1 \pm h)^{3}+\ldots
$$

it is not difficult to obtain

$$
\Phi\left(a_{i}^{2}, h\right)=\frac{1}{3} 2 a_{i}^{2} h=\frac{1}{3} \frac{\mu_{s} V_{i} H}{k T}
$$

which coincides with the expansion of the Langevin function when $2 a_{i}^{2} h=\mu_{s} V_{i} H / k T \ll 1$. Accidentally, it also follows from this that at any temperature differing from $T=0$ at $h \rightarrow 0$ the magnetization of each FM granule (and therefore of the whole GMM system) tends to zero.

Thus under these conditions, every granule of the GMM system and the system as a whole can be described by the classic Langevin theory, i.e., the phenomenon of superparamagnetism takes place.

The above expressions show that when the conditions $a_{i}^{2}<<1,2 a_{i}^{2} h \ll<1$ are implemented, the presence of the energy barrier in the GMM system granules is not essential (it can use the Langevin description).

\section{Conclusions}

It has been shown that, in a general case, real GMM systems with a magnetic anisotropic FM component cannot be described with the help of the Langevin classic theory, i.e., their thermodynamic equilibrium magnetization and static magnetic susceptibility cannot be described by the Langevin function and its derivatives.

The function has been found (substituting the Langevin function) which describes, in a universal way, the competition of full magnetic energy of a GMM system particle of any volume (without separating them into the so-called «blocked» and «unblocked») with their heat energy. It has been ascertained that the use of the concept of «blocking» is not necessary when the thermodynamic equilibrium properties of GMM are described. In addition to it, the use of such universal description for all the particles of a system and a system as a whole makes it possible to avoid such artificial and ambiguous models, one of which, for instance is used in [17].

The regions of changing external parameters $H$ and $T$ have been indicated (relating to internal parameters of an FM component of a GMM system) where classic Langevin description of investigated objects is possible. In GMM systems with a known function of granule distribution by their volume $f(V)$, magnetization curves $m(h)$ and curves of static magnetic susceptibility $\chi(h)$, registered at different temperatures, may be used both for determining the value of effective constant of magnetic anisotropy $K_{\text {eff }}$ and for clarifying the peculiarity of its temperature dependence. And conversely, the knowledge of the temperature dependence of the effective magnetic anisotropy constant $K_{\text {eff }}$ may allow one to reconstruct the volume distribution function $f(V)$ of the GMM system particles. The corresponding investigations of both the soft-magnetic and hardmagnetic ferromagnetic components of the different GMM systems will be published later.

\section{Aknowledgment}

The author would like to thank the Israel Ministry of Absorption (the Department of Science Absorption) for financial support, Prof. Gad Gorodetsky from the Ben-Gurion University of the Negev (Israel, Beer-Sheva) for the possibility of working in the region of magnetic properties of GMM systems and the extremely useful discussions and Dr. Victor Meerovich for the help in the work. 


\section{E. Stefanorskii}

1. S. V. Vonsovsky, Magnetism, Wiley, New York (1974).

2. C. P. Bean, and J. D. Livingston, J. Appl. Phys. 30, 1209 (1959).

3. I. S. Jacobs and C. P. Bean, Fine Particles, Thin Films and Exchange Anisotropy, in: Magnetism, v. III., G. T. Rado and H. Suhl (eds.), Academic Press (1963), p. 231.

4. B. D. Cullity, Introduction to Magnetic Materials, Addison-Wesley Publishing Company (1972).

5. Magnetic Properties of Fine Particles, J. L. Dormann and D. Fiorani (eds.), North Holland (1992).

6. A. E. Berkovitz, J. R. Mitchell, M. J. Carey, A. P. Young, S. Zhang, F. E. Spada, T. Parker, A. Hutten, and G. Thomas, Phys. Rev. Lett. 68, 3745 (1992).

7. J. Q. Xiao, J. S. Jiang, and C. L. Chien, Phys. Rev. Lett. 68, 3749 (1992).

8. C. P. Bean, J. Appl. Phys. 26, 1381 (1955).

9. L. Neel, Compt. Rend. 228, 664 (1949).

10. L. Neel, Ann. Geophys. 5, 99 (1949).
11. Yu. L. Reichel and M. I. Shliomis, Sov. Phys. JETP 40, 526 (1974)

12. V. V. Slezov, Theory of Diffusive Decomposition of Solid Solutions, Physics Review, Soviet Scientific Reviews/Section A, I. M. Khalatnikov (ed.), Harwood Academic Publishers, v. 17, part 3 (1995).

13. I. M. Lifshitz and V. V. Slyozov, J. Phys. Chem. Solids 19, 35 (1961).

14. M. H. Mathon, F. Maury, F. Dunstetter, N. Lorenzelli, C.-H de Novion, and F. Boue, J. Phys. (Paris) 3, 279 (1993).

15. F. G. West, J. Appl. Phys. 32, Suppl., 249S (1961).

16. Magnetism and Metallurgy, v. 1, A. E. Berkowitz and E. Kneller (eds.), Academic Press, NY and London (1969), p. 421.

17. B. Dieny, S. R. Teixeira, B. Rodmacq, C. Cowache, S. Auffret, O. Redon, and J. Pierre, JMMM 130, 197 (1994). 